\title{
Digitization of Operational Processes: Use Case of Standardization in an Assembly Learning Factory
}

\author{
Florian Magnani ${ }^{1,2(\bowtie)}$ \\ 1 Aix Marseilla Université, Université de Toulon, CERGAM, Aix-en-Provence, France \\ florian.magnani@centrale-marseille.fr \\ 2 Ecole Centrale de Marseille, Marseille, France
}

\begin{abstract}
The objective of this research is to set the ground for new research to measure the impact of Industry 4.0, by combining two types of analysis, that of the performance, and that of the human behaviors, using experiments with digital instruments. A preliminary test, then a first experiment focusing on standardization of operational processes are presented showing promising results to understand how operational and human performance are impacted by the Industry 4.0. This study serves as a first step to a larger research project about productivity measurement of Industry 4.0 transformation.
\end{abstract}

Keywords: Industry $4.0 \cdot$ Standardization $\cdot$ Behaviors $\cdot$ Productivity · Experiment

\section{Introduction}

Since the 1980s, the socio-technical and techno-economical environments have undergone profound transformations, leading enterprises to adopt numerous technological and organizational innovations. Research founds that these transformations performed successfully when they include the human dimension to the technical dimension associated with the chosen innovation. This human dimension is emphasized in all the studies dealing with the current industrial revolution named "Industry 4.0" [1] as a key element to positively influence the impact of these transformations on the enterprises' operational performance.

Study of technological innovations and its impact on performance have been a regular topic of interests. Thanks to fast improvements gathered by innovations, practices are constantly renewed, reshaped and put into perspective by the evolution of theoretical analysis. Today, given the gap between the expected performance and the real performance after the adoption of one of these innovations, it seems appropriate to study closely the characteristics of the so-called innovation with a focus on human behaviors. These behaviors studied throughout the utilization and appropriation of the innovation represent our units to explain this performance gap. 
The recent trend in research guide us in conducting experiments in an observational platform, relatively similar to an industrial environment, gathering qualitative and quantitative data. The objective of this research is to set the ground for new research to measure the impact of the current industrial revolution, by combining two types of analysis, that of the performance, and that of the human behaviors, using digital instruments. The first experiment based on a multidisciplinary approach focused on standardized work transformation in operation processes.

\section{State of the Art}

Before embarking on experimentation, the literature review aims to define how Industry 4.0 influenced operations digitization and indirectly the human behaviors.

Hermann et al. [2] define Industry 4.0 as "a collective term for technologies and concepts of value chain organization." Industry 4.0 seems to develop more intelligent, flexible, and dynamic factories by equipping manufacturing processes with sensors, actors, and autonomous systems. In addition, manufacturing processes have the capacity of fulfilling more complex and qualified standards and requirements of products, making value-added integration occurs horizontally and vertically. Regarding the digitization of manufacturing process, Liao et al. [3] depicted research areas focusing on more human aspects: (1) Safety and Security: to ensure that production facilities and products themselves do not pose a danger either to people or to the environment; (2) Work Organization and Design: to implement of a socio-technical approach for work organization and design to offer workers the opportunity to enjoy greater responsibility and enhance their personal development. In line with these recommendations, conducting research on digitization of manufacturing processes require a socio-technical perspective.

Contrary to conventional belief, automation does not lead to less human interaction or workerless production facilities; but the workers' competencies requirements may evolve. The evolution of workers' role in their new industrial system is related to their ability to take quick and efficient decision on production, orders... through intelligently processed data. In this application, workers' role in Industry 4.0 is the one of a decision maker in production planning and control - with the help of a decision support assistance system - or of a smart and enhancers operator [4]. Moreover, Liao et al. [3] recommendations make it clear the relationship with the employee's responsibility and autonomy. This refers also to the work focusing on the relationship between Lean practices and digitization of manufacturing processes [5]. Nevertheless, it exists few articles that discuss the adaptation of Jidoka principles, the one related to employee's empowerment [5] or its further benefits. So, the tension between the standardization of work and the participation of employees in Industry 4.0 environment remains a crucial point in the design of work organization.

In the current organizations, execution of the operational tasks is generally documented in a very descriptive and detailed way, thus forming a standardized work, the first step to standardization. This standardized work enables processes to be consolidated in a consistent, accurate and repeatable way, in order to reduce their variability and simultaneously improve their performance. Maginnis [6] demonstrates that standardized work improves team productivity and team learning. Standardized work is not 
only a method of documentation, but it also allows the analysis of work situations. This denotes the relationship between standardized work and problem solving that jointly promotes incremental improvements. That is to say, looking at Industry 4.0, the interaction between standardization, continuous improvement and technology enabling team learning and coordination needs to be explored. Research provides empirical evidence that developing employee participation regarding their standardized work, fostering learning and knowledge sharing at an organization level can obtain greater benefits from technologies adoption. According to this, our study focuses on human behaviors at an operational level.

At the end of the literature review, the emergent questioning is formulated as follows: when a technological or organizational innovation occurs, what is its impact from a technical and behavioral point of view on the operational performance?

\section{Methodology}

The following methodology respects part of the research agenda proposed by Liao et al. [3]. Previous research looking at the impact of innovations focused solely on performance or solely on human behaviors. These same researches display either qualitative or quantitative methodological positioning but rarely mixed. This study, combining quantitative and qualitative data, is therefore built on an experiment including a series of trial / error phases. The experimental environment is that of a learning factory, a dynamometer lamp assembly line with a student audience to reduce bias related to the experiences of professionals [6].

The observational platform is built on a training platform named DynEO, which offers trainings in operations management. The experiment was built on the existing environment while integrating new technologies and digital instruments to measure industrial and human performance. Direct observations on operators' behaviors helped in the analysis of the influence of changing practices. The observational lab includes non-invasive tools to reduce unwanted influence on behavior (biased observations). The lab is also flexible, easily adaptable to various circumstances for testing a wide variety of hypotheses. During the preliminary test, the following measurement equipment were used: Tobii Eye Tracker, FaceReader, the Observer.

After the preliminary test, eye-tracking glasses were chosen because it helps studying how people interact with their environment, what catches their attention, and what influences decision making. This technology records gaze, saccades and fixation data of workers, and thus enables insights about the thinking process. For the case study, saccades represented an indicator for the visual search (stress level) and fixation represents an indicator of cognitive processing (concentration level).

During the preliminary test, the efficiency between operators (experienced vs notexperienced) in the assembly of a lamp was differentiated. Both workers wore the eye tracking glasses, and their faces were filmed for real-time analysis of facial expressions. A camera was placed in front of the face of the operator, whereas a first scene camera recorded the global area of the workplace. A second scene camera integrated in the portable eye tracker recorded in detail the direction of the gaze of the participant. It was decided to manually code in The Observer the video from the Tobii glasses. Behaviors 
coded were 'task' (start, stop) and 'Regions of Interest' (main body, other components, tools, elsewhere).

The preliminary test helped us design the first experiment (Fig. 1). The experimental protocol was defined in conjunction with the performance and behavior factors to be measured. In our study on the use of standardized work, the differentiation between the handwritten standards (paper standard) used until now and the introduction of a technological innovation is materialized by the use of an interactive tablet on which the standard is available (digital standard). Experimentation related to the introduction of this digital standard was initially carried out on reference groups and will be extended over several test groups to ensure the validity and potential generalization of the results.
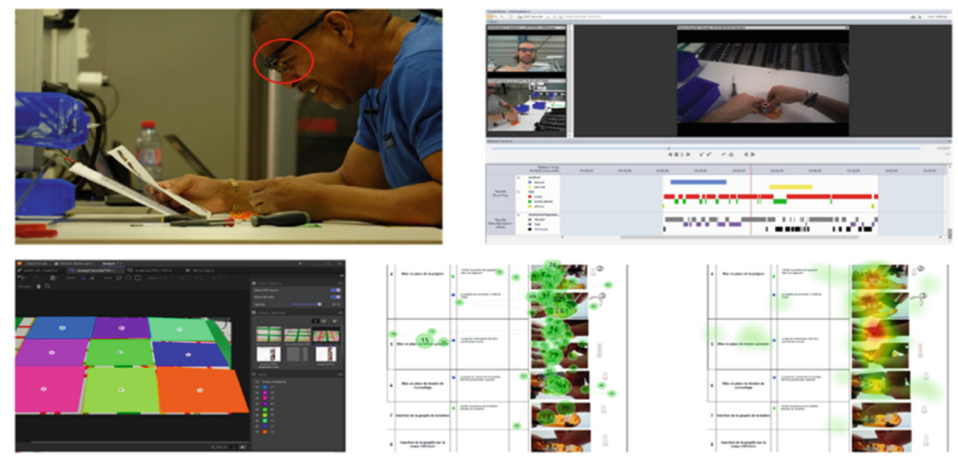

Fig. 1. Preliminary test and raw data.

\section{Results}

The preliminary test showed us that the students are using standardized work (SW) during their training and are mostly looking at visual components: $96 \%$ of their time is spend on looking at the visual/images included in the standard (Table 1). However, the comparison between the measured value of emotions and the one verbalized by students showed that measured value itself is not relevant. The variability of measured emotions throughout the preliminary test can be related to the one verbalized. So, the emotion variability can represent an indicator of work's impact on the student. Emotions will not be measured in the following experiment.

The results of the experimentation can be summarized as follows: in general, students use the standard support (paper and digital) during the training phase and sporadically during the second phase (quality). During the third phase (performance), students perform the task entirely from memory without consulting the support, which can have an impact on the sequence followed and the resulting quality (Table 2). The observations showed that students following the paper support come back to it when necessary and produce better lamps in terms of quality requirements. Conversely, students following the digital support have difficulty in delivering flawless lamps. The paper support seems to help identify quickly quality issues. 
Table 1. Comparative results from the preliminary test.

\begin{tabular}{l|l}
\hline Operator 1 & Operator 2 \\
\hline Experimented & Beginner \\
\hline Short execution & Long execution \\
\hline Stable emotions & Variable Emotions \\
High concentration on the body & $\begin{array}{l}\text { High concentration on components } \\
\text { Stable Heart Rate }\end{array}$ \\
\hline
\end{tabular}

Student's perception was gathered throughout a survey: all consider that the tasks require a strong and permanent concentration. Students prefer the paper standard which gives them more flexibility. They showed less signs of incomprehension and seemed more focused. Depending on the standard used, a variation in the perception of the necessary time to achieve the task was noticeable (too short for those who use the digital standard). Those who used the digital standard questioned the given autonomy when they identified a problem to solve. If they identify a situation not foreseen by the standard, they find themselves stuck and call for outside help.

Table 2. Comparative data from paper standard and the digital standard utilization.

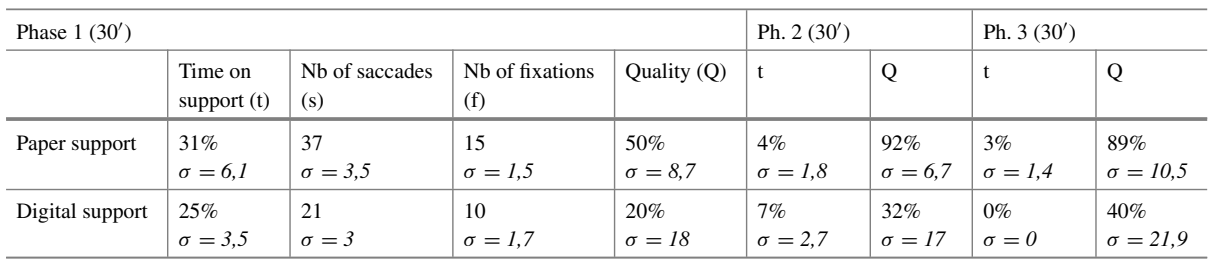

In the end, these three phases enabled us to observe a real difference in training, and therefore in performance according to the standards used. Because of its speed constraints, the digital standard does not attract students' attention during the key phases of learning. Conversely, the paper standard ensures a quality training, but slower. Finally, the paper standard is open to more criticisms or way to improve it which seems to foster student creativity while improving knowledge of the tasks performed. A next step would be to mix the utilization of digital and paper support according to the training and executing phases.

This experiment had some limitations: the sample was homogenous but small (about twenty students). Some of the data should be used with caution as some are based on subjective judgments (video coding, perception evaluated by a survey), on the capacity of each individual to follow a standard and the associated reactions (even if data were triangulated to limit the subjectivity). The results can vary greatly from one individual to another. It will be necessary to repeat the experiment on a larger sample. In addition, the measurement of emotions through image processing software can provide a better exhaustiveness of human behaviors interpretations. 


\section{Conclusion}

This study serves as a first step to a larger research project about productivity measurement of Industry 4.0 transformation. The results showed promising data at the crossroad between performance and human behaviors. There are currently not a lot of studies about eye-tracking glasses technology applied in manufacturing environment, but the benefits of this technology are becoming a stimulating topic for researchers. A question remains unanswered: is the digital standardization about automate the management of work or automate the work itself?

\section{References}

1. The Boston Consulting Group: “The Most Innovative Companies 2018 : Innovators go all in on digital" (2018)

2. Hermann, M., Pentek, T., Otto, B.: Design principles for Industry 4.0 scenarios. In: Proceedings of the 49th Hawaii International Conference on System Sciences, pp. 3928-3937 (2016)

3. Liao, Y., Deschamps, F., de Loures, E.F.R., Ramos, L.F.P.: Past, present and future of Industry 4.0 - a systematic literature review and research agenda proposal. Int. J. Prod. Res. 55(12), 3609-3629, June 2017

4. Longo, F., Nicoletti, L., Padovano, A.: Smart operators in industry 4.0: a human-centered approach to enhance operators' capabilities and competencies within the new smart factory context. Comput. Ind. Eng. 113 (2017)

5. Rosin, F., Forget, P., Lamouri, S., Pellerin, R.: Impacts of Industry 4.0 technologies on Lean principles. Int. J. Prod. Res. 58, 18 (2019)

6. Maginnis, M.A.: The impact of standardization and systematic problem solving on team member learning and its implications for developing sustainable continuous improvement capabilities. J. Enterprise Transform. 3(3), 187-210 (2013)

Open Access This chapter is licensed under the terms of the Creative Commons Attribution 4.0 International License (http://creativecommons.org/licenses/by/4.0/), which permits use, sharing, adaptation, distribution and reproduction in any medium or format, as long as you give appropriate credit to the original author(s) and the source, provide a link to the Creative Commons license and indicate if changes were made.

The images or other third party material in this chapter are included in the chapter's Creative Commons license, unless indicated otherwise in a credit line to the material. If material is not included in the chapter's Creative Commons license and your intended use is not permitted by statutory regulation or exceeds the permitted use, you will need to obtain permission directly from the copyright holder.

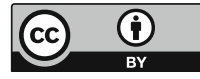

